\title{
Multichannel Raman polarizer with suppressed relative intensity noise for wavelength division multiplexing transmission lines
}

\author{
Victor V. Kozlov, ${ }^{1,2, *}$ Javier Nuño, ${ }^{3}$ Juan Diego Ania-Castañón, ${ }^{3}$ and Stefan Wabnitz ${ }^{1}$ \\ ${ }^{1}$ Department of Information Engineering, Università di Brescia, Via Branze 38, 25123 Brescia, Italy \\ ${ }^{2}$ Department of Physics, St.-Petersburg State University, Petrodvoretz, St.-Petersburg 198504, Russia \\ ${ }^{3}$ Instituto de Optica, Consejo Superior de Investigaciones Cientificas (CSIC), 28006 Madrid, Spain \\ ${ }^{*}$ Corresponding author: victor.kozlov@ing.unibs.it
}

Received November 21, 2011; revised March 1, 2012; accepted March 6, 2012;

posted March 15, 2012 (Doc. ID 158487); published May 30, 2012

\begin{abstract}
We propose a method of suppressing the relative intensity noise caused by polarization-dependent gain that is inherent to Raman polarizers (RPs). This method involves bit-synchronously scrambling the state of polarization of a pulse (bit) before the pulse enters the RP. The proposed solution works for RPs operating in a depleted regime and is compatible with multichannel configurations. (C) 2012 Optical Society of America

OCIS codes: $230.5440,230.1150,230.4320$.
\end{abstract}

Raman polarizers (RPs) were first proposed and experimentally demonstrated in the pioneering paper by Martinelli et al. in [1]. There, an RP was realized by using a few-kilometer-long span of telecommunication ultralow polarization mode dispersion (PMD) fiber pumped by a few-watt power beam. A weak signal beam copropagating along the fiber together with the pump was efficiently repolarized, i.e., acquired one and the same state of polarization (SOP) toward the output of the polarizer regardless its SOP set at the input: the signal SOP was attracted toward the pump SOP. As it was theoretically proven later, RPs can work not only in such copropagating geometry $[2,3]$, but also in a counterpropagating configuration $[4,5]$.

The repolarization process (attraction) takes place due to the highly polarization-dependent gain (PDG) inherent to silica: the Raman gain coefficient along the pump SOP takes its maximum value $g$, while the gain of the orthogonal component is virtually zero. Along with the obvious advantage of its potential applications in repolarization and enhanced gain, PDG brings also a drawback-the repolarized beam acquires a high level of relative intensity noise (RIN), even if the initial unpolarized light was steady in time intensity. This RIN is directly caused by PDG and should not be mistaken for other forms of RIN arising, for example, from amplitude noise in the pump beam.

One possible way to suppress this RIN is to operate the $\mathrm{RP}$ in the depleted regime, as suggested in [6]. However, this method has limitations when applied to telecom systems: (i) it becomes ineffective for a pulse stream with low extinction ratio, because "zeros" are amplified in the undepleted regime and therefore experience stronger gain than "ones," which are amplified in the depleted regime, finally resulting in a severe degradation of the extinction ratio; (ii) while working perfectly for one channel, the method is not scalable to multichannel (WDM) operation, when the competition for gain of temporally overlapping pulses from different channels introduces its own RIN into each channel; (iii) the RIN ratio $R$, defined later and which characterizes the gap between the maximally and the minimally amplified "one," is very high, thereby pointing to the inevitable (though maybe rare) failure in device performance.

In this Letter we propose an alternative method for combating RIN in RPs for WDM signals, which is free of the three above-listed limitations. The method is conceptually similar to the proposal of $[\underline{7}, \underline{8}]$, where a different two-section fiber configuration was used in the single-channel case.

The main idea here is to scramble the SOP of each bit prior to launching it into the RP, as shown in the scheme of Fig. 1. Indeed, electro-optic polarization scramblers may provide bit-synchronous modulation of the initial SOP of the pulse in such a way that the SOP of the pulse leaving the scrambler undergoes a long excursion over the Poincaré sphere across its time width [9]. In this
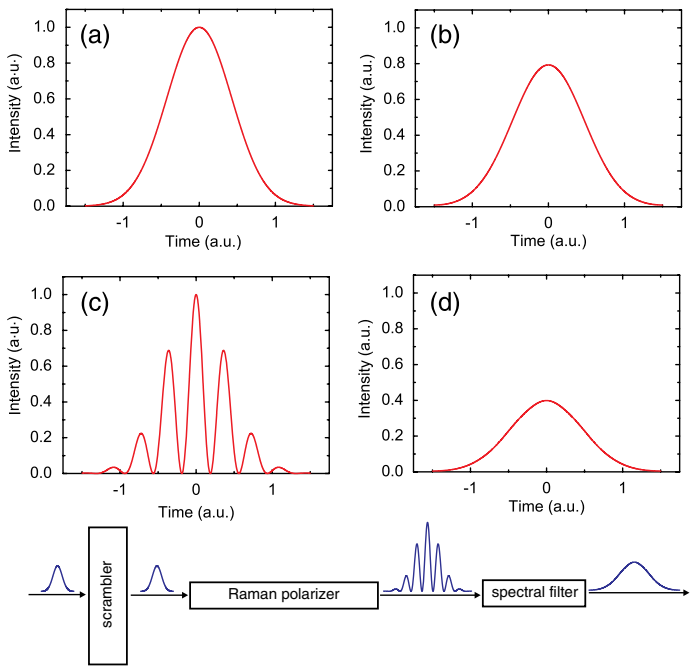

Fig. 1. (Color online) Pulse from a signal beam (a), (b) before and (c), (d) after the RP. (a) Input shape-Gaussian; (b) input shape under the action of the spectral filter (note that the spectral filter is not present in the scheme at the input, and this shape is solely shown to illustrate the action of the filter on the Gaussian pulse); (c) outcoming pulse shape immediately after the RP; (d) pulse after spectral filtering of the shape shown in (c). A sketch of the setup is shown at the bottom. 
way we may bring each pulse from the sequence into a highly unpolarized state. At first sight it might seem counterintuitive that, in order to get a fully polarized light out of the device, we first need to depolarize it as strongly as possible. However, we are going to demonstrate shortly that such a polarization prescrambling indeed leads to a significant PDG-induced RIN suppression even for WDM signals.

We shall consider the model of an ideal copropagating multichannel RP, which is the extension of our previous one-channel model [2] to the case of multichannel operation. We are interested in the limit of vanishing PMD, when the RP performs its function at its best [2-5]. In this limit the randomly birefringent telecom fiber turns into a perfectly isotropic fiber. The equations of motion for the signal and pump beams read as

$$
\begin{aligned}
\left(\partial_{z}-v_{i}^{(s)} \partial_{t}\right) \mathbf{S}_{i}^{(s)}= & \bar{\gamma} \mathbf{S}_{i}^{(s)} \times\left(\mathbf{S}^{(p)}+\sum_{j=1, \neq i}^{N} \mathbf{S}_{j}^{(s)}\right) \\
& +(g / 2)\left(S_{0}^{(p)} \mathbf{S}_{i}^{(s)}+S_{0 i}^{(s)} \mathbf{S}^{(p)}\right)-\alpha \mathbf{S}_{i}^{(s)}, \quad(1) \\
\left(\partial_{z}-v^{(p)} \partial_{t}\right) \mathbf{S}^{(p)}= & \bar{\gamma} \mathbf{S}^{(p)} \times \sum_{j=1}^{N} \mathbf{S}_{j}^{(s)} \\
& +(g / 2) \sum_{j=1}^{N} \epsilon_{j}^{(p)}\left(S_{0}^{(p)} \mathbf{S}_{j}^{(s)}+\mathbf{S}^{(p)} S_{0 j}^{(s)}\right)-\alpha \mathbf{S}^{(p)},
\end{aligned}
$$

where $i, j$ label the channel number in the $N$-channel RP; $\mathbf{S}_{i}^{(s)}=\left(S_{1}^{(s)}, S_{2}^{(s)}, S_{3}^{(s)}\right)\left[\mathbf{S}^{(p)}=\left(S_{1}^{(p)}, S_{2}^{(p)}, S_{3}^{(p)}\right)\right]$ is the Stokes vector of the signal (pump) beam, $S_{0 i}^{(s)}\left[S_{0}^{(p)}\right]$ is the signal (pump) power; $v_{i}^{(s)}\left[v^{(p)}\right]$ is the group velocity of the signal (pump) beam; $\bar{\gamma}=\frac{8}{9} \gamma, \gamma$ is the Kerr coefficient; $\alpha$ is linear fiber loss; and $\epsilon_{i}^{(p)}=-\lambda^{(p)} / \lambda_{i}^{(s)}, \lambda_{i}^{(s)}\left[\lambda^{(p)}\right]$ is the signal (pump) wavelength. The symmetry of Eqs. (1) and (2) implies that the performance of the RP is independent of a particular choice of the pump SOP, which we now fix by letting $S_{1}^{(p)}(0)=S_{0}^{(p)}(0)$. The pump is a CW with no inherent amplitude noise.

For quantifying the level of RIN, we introduce the RIN ratio $R_{i}$ and the normalized root-mean-square deviation (RMSD) of peak intensities. For the $i$ th channel with $i=1, \ldots, N$, one has $\mathrm{CV}(\mathrm{RMSD})_{i}=\langle I\rangle^{-1} \sqrt{\left\langle(I-\langle I\rangle)^{2}\right\rangle}$ (here $\mathrm{CV}$ is the coefficient of variation), where $I_{j}=$ $\max _{j}\left[S_{0}^{(s)}(L)\right]$ is the peak intensity of a pulse in the $j$ th "one" bit, and the average $\langle I\rangle \equiv M^{-1} \sum_{j=1}^{M} I_{j}$ is taken over all "one" bits in the sequence. In our example we use a pseudo-random sequence of 512 bits with $M=256$. The smaller the CV(RMSD), the less pronounced the intensity fluctuations and the better the performance of the RP. Nevertheless CV(RMSD) only characterizes the statistical properties of the device, as it tells us nothing about the occurrence of rare events. In this respect the RIN ratio $R$ is more helpful. For evaluating this quantity, we need to scan over all possible transmitted bits and take the absolute maximum and absolute minimum of the peak intensity of the "one" bits. As an estimate, we can say that, when $R$ is as high as $E^{-1}$, where $E$ is the extinction ratio, then some events happen in which the output intensity of the "one" bit becomes comparable to the average output intensity of a "zero" bit, and a failure occurs, because of the indistinguishability of this "one" from a "zero." Overall, it is desirable to get $R$ as close to unity as possible. We shall present shortly an analytic estimate showing that, for an RP in the undepleted regime without prescrambling, $R$ is as high as $G$, where $G$ is linear gain of the polarizer, while it is only three for the same setup with prescrambling.

Let us define the input (at $z=0$ ) SOP of a "one" bit of the signal beam in the Stokes space as

$$
\begin{aligned}
& S_{1}^{(s)}=S_{0}^{(s)}(t, 0) \sin \left[\phi_{1}+\omega(t)\right] \cos \left[\phi_{2}+\omega(t)\right], \\
& S_{2}^{(s)}=S_{0}^{(s)}(t, 0) \sin \left[\phi_{1}+\omega(t)\right] \sin \left[\phi_{2}+\omega(t)\right], \\
& S_{3}^{(s)}=S_{0}^{(s)}(t, 0) \cos \left[\phi_{1}+\omega(t)\right] .
\end{aligned}
$$

The shape of $S_{0}^{(s)}(t, 0)$ is Gaussian. Phases $\phi_{1}$ and $\phi_{2}$ are constant across each bit slot but differ from slot to slot. The modulation $\omega(t)$ describes the action of the polarization scrambler. We choose $\omega(t)=8 \pi t / T$, where $T$ is the bit duration. Excessive scrambling speeds should be avoided as they may lead to the spectral overlap of neighboring channels. We vary $\phi_{1}$ and $\phi_{2}$ from bit to bit in such a way that the overall degree of polarization (DOP) of our 512 bit long sequence at the input is as small as 0.02 even in the absence of the scrambler, thus representing a case of unpolarized light. At the output of the RP, whose parameters are listed in the caption of Fig. 2 , the DOP becomes as large as 0.997 in all simulation runs presented in this Letter. This observation indicates that the RP perfectly performs its main function-the repolarization of light.
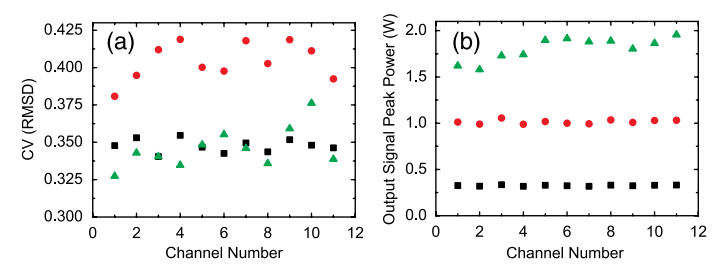

Fig. 2. (Color online) 11-channel $\mathrm{RP}$ in three regimes: (a) $\mathrm{CV}(\mathrm{RMSD})$ and (b) average output signal peak power of "one" bits, both versus channel number. Undepleted regime with no walk-off (black squares), depleted regime with no walk-off (red circles), and depleted regime with walk-off (green triangles). Parameters are $\gamma=1(\mathrm{~W} \cdot \mathrm{km})^{-1} ; g=0.6(\mathrm{~W} \cdot \mathrm{km})^{-1}$; $\alpha=0.2 \mathrm{~dB} / \mathrm{km} ; P=8 \mathrm{~W}$; input signal power, $1 \mathrm{~mW}(10 \mathrm{~mW})$ in the undepleted (depleted) regime; fiber length, $L=1.5 \mathrm{~km}$; $N=11 ; \lambda^{(s)}=1.55 \mu \mathrm{m} ; \lambda^{(p)}=1.45 \mu \mathrm{m}$; the pulse shape is Gaussian with $\mathrm{FWHM}=8.33 \mathrm{ps}$; duty cycle 0.33 ; bit slot $25 \mathrm{ps}$; $\left[v^{(s)}\right]_{j}^{-1}=\left[v^{(p)}\right]^{-1}+\Delta \beta j, j=1, \ldots, 11$, with $\Delta \beta=0$ for no walkoff regime, $\Delta \beta=2.4 \mathrm{ps} / \mathrm{km}$ for regime with walk-off. The spectral filter is modeled as $\exp \left[(-1 / 2) \omega^{2} T_{f}^{2}\right]$ with $T_{f}=2.1 \mathrm{ps}$. All points are obtained with prescrambling. 
Let us solve Eqs. (1) and (2) in the regime of undepleted pump, in the absence of linear losses, and neglecting nonlinear cross-polarization modulation effects (i.e., setting $\bar{\gamma}=0$ ). In this regime all channels are equivalent, and for each channel we can write $S_{1}^{(s)}(L)=\frac{1}{2}(G+1) S_{1}^{(s)}(0)+\frac{1}{2} S_{0}^{(s)}(0)(G-1) 4$, where $G=$ $\exp (g P L)$ with $P=S_{0}^{(p)}(0)$ as the pump power. Note that we write down only the solution for $S_{1}^{(s)}$, as it is this component of the signal Stokes vector that largely dominates at the output [the other two components are not of interest to us because they grow much slower, i.e., $\propto \exp (g P L / 2)]$.

In the absence of prescrambling, the SOP does not change across the bit, and it is easy to find both maximum and minimum for $\left|S_{1}^{(s)}(L)\right| \approx S_{0}^{(s)}(L)$ : $\max \left[S_{1}^{(s)}(L)\right]=$ $G S_{0}^{(s)}(0)$, and $\min \left[S_{1}^{(s)}(L)\right]=-S_{0}^{(s)}(0)$. So $R=G$. The maximum is realized when the input signal SOP is aligned with the pump SOP, while the minimum occurs when these two SOPs are orthogonal.

In presence of prescrambling, the SOP evaluation becomes involved. Moreover, the value of $R$ starts to depend on the width of the spectral filter and on the specific way how the scrambling is implemented. For our choice of parameters, we numerically scanned over all $\phi_{1}$ and $\phi_{2}$ and obtained $\max \left[S_{1}^{(s)}(L)\right] \approx 0.6 G S_{0}^{(s)}(0)$ and $\min \left[S_{1}^{(s)}(L)\right] \approx 0.2 G S_{0}^{(s)}(0)$ for $G \gg 1$, resulting in $R=3$. This result means that the peak intensity of the output signal never drops below 50\% and never jumps above $50 \%$ with respect to its average level.

More information about the statistical characteristics of the RP can be obtained via simulation of the average peak intensity and the CV(RMSD). The results, numerically obtained by solving Eqs. (1) and (2), are shown in Fig. 2. As a reference, we may note that the $\mathrm{CV}(\mathrm{RMS} \overline{\mathrm{D}})=0.456$ (average over all channels) for the undepleted pump regime without prescrambling. When the prescrambling is introduced, the CV(RMSD) drops down to 0.341 (average over all channels); see Fig. 2. Therefore, intensity fluctuations are significantly suppressed by the bit-synchronous polarization scrambler. This leads to a corresponding decrease of the bit error rate (BER) of a pseudo-random bit sequence when prescrambling is used. As an example, we consider the signal in the first channel out of an 11-channel WDM comb with an optical-signal-to-noise ratio of $20 \mathrm{~dB}$. By feeding this signal into a direct detection receiver using the Chi2 estimation method with intersymbol interference correction, we numerically estimated that the BER at the output decreases from $7.2 \times 10^{-4}$ down to $2.6 \times 10^{-5}$ when prescrambling is applied. Thus, the benefits of amplitude jitter reduction due to prescrambling overcome the negative impact of the additional timing jitter that is introduced by the technique.

In the depleted pump regime, the amount of the group velocity dispersion-induced walk-off (understood here as the dependence of the group velocity on channel number/wavelength) starts to play an important role. In the example shown in Fig. 2 , the average peak power almost doubles in the presence of walk-off when compared to the case of no walk-off. The walk-off suppression of the competition of channels for the gain is reflected in the CV(RMSD), which remains at the minimum level [0.346, obtained by averaging the CV(RMSD) over all channels]. This observation should be compared to the performance of the same RP, but without prescrambling. In this case in the depleted regime $\mathrm{CV}(\mathrm{RMSD})=0.443$ with walk-off and 0.488 with no walk-off, where both values are obtained by averaging over all channels.

In conclusion, we have suggested to use a bitsynchronous polarization scrambler prior to an $\mathrm{RP}$ with the goal of suppressing the intensity fluctuations of the outcoming stream of pulses. A spectral filter should follow the RP in order to smoothen strong temporal modulation acquired by the pulse while passing through the RP. The performance has been quantified in terms of the RIN ratio, CV(RMSD), and BER. We numerically verified that the technique is also applicable to the nonreturn-to-zero modulation format.

This work was carried out with support from the Italian Ministry of University and Research (MIUR) in the framework of the "Scientific Research Project of Relevant National Interest" (PRIN 2008) entitled "Nonlinear cross-polarization interactions in photonic devices and systems" (POLARIZON), the research projects TEC2008-05791 and TEC2011-27314 from the Spanish Ministry of Science and Innovation, and the 2009 ItalySpain integrated action "Nonlinear Optical Systems and Devices" (HI2008-0075). We used VPI TransmissionMaker 8.6 for the numerical estimation of the received BER.

\section{References}

1. M. Martinelli, M. Cirigliano, M. Ferrario, L. Marazzi, and P. Martelli, Opt. Express 17, 947 (2009).

2. V. V. Kozlov, J. Nuño, J. D. Ania-Castañón, and S. Wabnitz, Opt. Lett. 35, 3970 (2010).

3. L. Ursini, M. Santagiustina, and L. Palmieri, IEEE Photon. Technol. Lett. 23, 254 (2011).

4. V. V. Kozlov, J. Nuño, J. D. Ania-Castañón, and S. Wabnitz, J. Lightwave Technol. 29, 341 (2011).

5. F. Chiarello, L. Ursini, L. Palmieri, and M. Santagiustina, IEEE Photon. Technol. Lett. 23, 1457 (2011).

6. V. V. Kozlov and S. Wabnitz, IEEE Photon. Technol. Lett. 23, 1088 (2011).

7. S. Sergeyev, S. Popov, and A. T. Friberg, Opt. Express 16, 14380 (2008).

8. S. Sergeyev and S. Popov, IEEE J. Quantum Electron. 48, 56 (2012).

9. F. Heismann, D. A. Gray, B. H. Lee, and R. W. Smith, IEEE Photon. Technol. Lett. 6, 1156 (1994). 